\title{
Magic of Language
}

\author{
Bernard Korzeniewski \\ Faculty of Biochemistry, Biophysics and Biotechnology, Jagiellonian University, Kraków, Poland \\ Email: bernard.korzeniewski@gmail.com
}

Received July 23 $3^{\text {rd }}, 2013$; revised August 23 $3^{\text {rd }}, 2013$; accepted August $30^{\text {th }}, 2013$

Copyright (C) 2013 Bernard Korzeniewski. This is an open access article distributed under the Creative Commons Attribution License, which permits unrestricted use, distribution, and reproduction in any medium, provided the original work is properly cited.

\begin{abstract}
Language, through the discrete nature of linguistic names and strictly determined grammatical rules, creates absolute, "quantized", sharply separated "facts" within the external world that is continuous, "fuzzy" and relational in its essence. Therefore, it is similar, in some important sense, to magic, which attributes causal and creative power to magical words and formulas. On the one hand, language increases greatly the effectiveness of the processes of thinking and interpersonal communication, yet, on the other hand, it determines and distorts to a large extent the picture of the world created within the mind. The relatively smallest (but still significant) magical admixture is present in science, because of its relatively reliable methodology, while the largest is found in religion and a large part of philosophy. The magical nature of language also manifests itself in logic and mathematics that refer to ill determined, fuzzy objects, sets and relations in the real world. The meaning of linguistic names is based on the conceptual network-an epiphenomenon (continuous in its essence) of the neural network - where interactions between particular concepts are based on the relation of connotation. The names and formulas of language correspond to these concepts which are best separated and determined. A direct relation of denotation between the elements of language and "facts" of the world is an illusion. While we cannot dispense with language because of its immense usefulness, we must remember about its "fact-creating" nature and influence on our thought and cognitive processes. The picture of the reality created as the result of them is to a large extent formed and deformed by the nature of language, and not by the "immanent" properties of the world in itself.
\end{abstract}

Keywords: Language; Cognitive Science; Neurophysiology; Self-Consciousness; Conceptual Network

\section{Introduction}

Speaking shortly and in somewhat simplified terms, the faith in magic consists in the belief that some appropriately chosen formulas of language (words, sentences) and, more broadly, various signs, symbols, numbers and activities (including gestures, graphical signs as well as complex rituals and ceremonies) possess causal power in the real, material (but also psychical) world. In accordance with this belief, magical formulas can cause objects shift, provoke a lightning or induce an illness in a human or an animal. Puncturing an enemy's puppet with splinters is supposed to cause his death. Occult signs placed on walls or doors protect against evil demons, while enchantment is able to change the emotional state of the enchanted person. Generally, "casting spells" brings into existence (or annihilates) various sorts of entities, phenomena, objects and processes. While knowledge (e.g., common or scientific) says something (by definition) about the real world, constitutes more or less adequate picture of the objective reality, magic "creates" a new reality that has nothing (or almost nothing) to do with the "true" one. Of course, magic creates entities only seemingly, in the opinion of persons believing in its power.

The thesis of the present article is this that not only strictly magical spells and formulas, but also - in some more general epistemological sense - the whole language plays in its essence the role of magic in the process of cognition of the reality surrounding us. Language is to describe the world, to serve the purpose of communicating about that world. However, through its nature (discrete names and rigorous grammar), it co-creates the picture of this world and, in some disciplines, it completely dominates this picture. Thus, as magic was to bring various entities and phenomena into existence, so language "brings into existence" sharp, "quantized" facts and relations within the world that is continuous in its essence, and it frequently does so without (almost) any reference to this world. Additionallyaccording to the Sapir and Whorf hypothesis (Sapir, 1921; Whorf, 1940; Kay \& Kempton, 1984)—particular ethnical languages shape the picture of the reality through their grammatical structure (but also vocabulary) in somewhat (sometimes very) different ways. For this reason, such pictures may to a large extent not adhere to each other. The impossibility of perfect translation of two ethnic languages into each other (the so-called indeterminacy of translation) (Quine, 1960) seems in its essence, after some pondering, trivially true. Of course, the peculiarity - being a derivative of upbringing in a such-andnot-another culture (of which ethnical language is a part), and also individual (inborn and acquired) properties of the brain and mind of a given person - superimposes on the peculiarity of the world picture caused by the specificity of an ethnic language. The ability to acquire language during individual life is inborn in humans. There are special centers in the human brain, namely Broca's area and Wernicke's area, that are responsible for generating speech (expressing thoughts in words) and un- 
derstanding language, respectively. Along these lines, Chomsky formulated his Universal Grammar theory and postulated that the ability to learn grammar is hard-wired into the brain (Chomsky, 1968). However, any particular grammar and vocabulary are learned during individual life.

However, in the present article the stress will be put on something else. It was practically always being (silently) assumed that words and sentences of language correspond to various objects, sets, categories, entities, aspects and processes of the world. This thesis was most explicitly formulated by (early) Wittgenstein (Wittgenstein, 1921), who claimed that language refers (or at least should refer) to the facts of the world. Sentences of language that do not refer (correspond) clearly and univocally to these facts were eliminated by Wittgenstein as senseless. This philosopher-and also neo-positivists who sympathized with him (especially from the Viener circle) - wanted to base all rational knowledge, especially science, on names and sentences that remain in direct correspondence with the facts of the world. Leaving aside the fact that this approach rejected most scientific theories (and practically the entire philosophy) as unacceptable, nobody managed to define clearly what "facts of the world" are. In "Tractatus logicophilosophicus" Wittgenstein did not resolve this problem, but put it off - as relatively unimportant - for the future.

Such a zero-or-one division of linguistic sentences into those corresponding with facts of the world and those having nothing in common with these facts, I regard as enormous mistakes. The real state of affairs looks quite differently. Objects, aspects and phenomena of the world (and so, generally speaking, the discussed "facts") described by words and sentences of language do not in reality exist at all in an autonomous and absolute way. Therefore, if one says that he/she ate today sushi for a dinner, that dinosaurs became extinct over 65 million years ago or that the force of gravity acts between bodies endowed with mass, then one does not express sentences about facts that are true or false (or objectively "existing" or "non-existing") in an unconditional way, but one performs some rough and approximate description of reality that is continuous in its essence. This thesis can seem absurd at first glance, but I will try to demonstrate that arguments supporting it are irresistible.

There exists some small superficial similarity between my idea and Austin's speech acts and "performatives" (Austin 1962). However, this theory concerned mostly social "facts" and assumed anyway that facts in themselves exist in the external world. Frege postulated in his mediated reference theory (Frege, 1960) that the meaning of a name cannot be simply reduced to the object which it refers to. For instance, the same object can have two different names, with different semantic connotations. However, this proposal, again, has little to do with the content of the present paper and accepts objectively existing absolute "facts". Generally, as far as I know, the main concepts I formulate below have not been published before elsewhere, at least not in so extreme a formulation. Of course, it is widely recognized that many predicates of linguistic names are vague (compare for instance, the paradox of the heap). However, I argue that, on principle, all such predicates ("facts of the world") are at best vague (not well determined), and at worst, they do not exist at all. Perhaps a point of view closest to my ideas was proposed by Bertrand Russell (Russell, 1923) ${ }^{1}$, although it seems that he attributed vagueness rather to linguis-

${ }^{1}$ I found and read (with great pleasure) this article when I was finishing the present article, so I came to some similar conclusions independently. tic representations of things than to "things in themselves". This is opposite to what I think.

\section{Language and Facts-Individual Person}

Let us reflect on the facts of the world described by words and sentences of language. For a start, let us consider some concrete person, for instance mister XY. Does this individual (it could be Julius Cesar, already deceased, frequently used by logicians and philosophers) exist (or did he exist) in somehow absolute and unconditional way (and therefore constitutes/constituted a sharply and clearly separated "fact of the world")? What does in fact its "existence", "identity" consist in? Let us begin with the matter it is built of, i.e. a given set of concrete atoms. It is well known that, in contrast to (most) inanimate objects, living organisms (of course including humans) unceasingly keep replacing the substance of their bodies. Biological systems are dissipative structures (Prigogine, 1980; Prigogine $\&$ Stengers, 1984) - their existence and functioning is driven and conditioned by a continuous flow of matter, energy and information. During weeks and months, the atoms that the human body (in particular its cells) is composed of are excreted from it, and, in return, new organic components of the body are built from the matter taken up as food (this is one of the two reasons, why organisms that do not grow must feed; the other reason is gaining energy as a result of combustion of assimilated organic compounds with the participation of oxygen - this is why humans must respire). The enamel of teeth and mineral components of bones constitute an exception here. Therefore, it is certainly not given concrete material components that decide about the identity of mister XY. However, undoubtedly, despite the continuous exchange of matter, the atoms entering into composition of the human body adopt a certain form, maintain a given concrete structure, that is a mutual arrangement of atoms of various elements (and of body components on higher levels of the hierarchy of complexity, such as macromolecules, cells, tissues and organs). However, the problem consists in the fact that this structure evolves with age, from the stage of the fertilized egg cell (which conventionally is regarded as the first stage of embryonic development) to natural death. Therefore, one must weaken this criterion of identity of a given person: it is not a concrete form that matters here, but rather a continuity of changes of this form in time. In this interpretation, mister $\mathrm{XY}$ would constitute some sequence of forms transforming themselves one into another during his lifetime, a kind of an ice block "suspended" in four-dimensional space-time. However, we face here another problem. Namely, what will happen if we start to decompose mister XY into parts? Let us begin innocently with cutting off his hair and nails. Of course, we will still think that we deal with our XY. Even a much more drastic amputation of a limb will not, in our conviction, take the identity from XY. The same can be said about an amputation of further limbs, or transplantation of internal organs coming from other persons, or even from animals (e.g. a pig's heart). Even if we removed or "exchanged" the majority of the XY's body in this way, most people would think that this is still XY (although this is maybe already not so obvious). Let us assume, however, that we are able to isolate (without damaging it) and maintain alive the "naked" brain of our unfortunate "patient". Is this still $\mathrm{XY}$ ? Does there exist at all a good answer to such a question? In fact, the answer does not have any important significance in this context. To explain this, let us start again with intact $\mathrm{XY}$ and remove one atom from his body. Undoubtedly, the result 
will be still XY. Of course, the same will happen, when we do this with two, three or four atoms. Yet in the end, following this procedure with sufficient consequence, we can fully decompose $\mathrm{XY}$ into atoms in this way. Even if we leave the isolated brain of the victim of our semantic analysis for the end, the gradual disintegration will finally affect this organ as well. And therefore, at a certain moment, after taking away a proper number of atoms, a removal of a subsequent atom will lead to the transformation of XY into not-XY. It is unimportant where we will, arbitrarily, mark out this border. In each case, it will look fully artificial. After all, before taking away the next atom, this still-XY differs much more from the initial XY than from the already-not-XY that comes into being after taking away that atom. This is just the magic of language-imposing discrete, apparently perfectly determined and separated "facts", such as "XY", onto the world that is continuous in its essence-that forces us to face this sort of paradoxes. Of course, when we reach his brain during the decomposition of $\mathrm{XY}$ into atoms, when subsequent neural connections and neurons begin to vanish, the psyche of XY will also gradually vanish. This will not be a sudden act of disappearance of the "soul". Human consciousness can undergo splitting into two consciousnesses, almost completely independent of each other, when the operation of commissurotomy is carried out (i.e. cutting of corpus callosum that connects the two brain hemispheres), which was once used to cure epilepsy. Large character changes (different, but generally consisting in mental impoverishment) can occur as a result of damages of various brain parts, chiefly frontal and prefrontal cortex (damage of other parts handicaps various cognitive functions). Alcoholism and drug addiction, associated with creeping degeneration of different brain areas, lead to gradual degeneration of personality. A similar general process occurs in Alzheimer and Parkinson diseases or, unfortunately, at advanced age. Due to taking away atom by atom from the brain, the transition between fully expressed consciousness and psychical nothingness will be continuous, "smooth" and hardly noticeable, as fading of colors on an old photograph. Anyway, as in the case of the structure (form) of the body, the "content" of psyche changes during the entire human life. However, unlike the form of the body, the mind can undergo a sudden shift to a completely different course, when the light of consciousness (directed by the phenomenon of attention) unexpectedly takes in still new and new "mental objects", jumps from one thought to another (or from one sensation to another), although the "general potential psyche"- that is broadly understood personal properties-undoubtedly evolves at a much slower rate. Anyway, there exists immanent discontinuity in the existence of consciousness in the state of dream, especially its deep phase.

Instead of decomposing XY into atoms, we can graduallyproceeding in very tiny steps - change its structure and function in such a way that we ultimately transform him into another man (let us call him XY2) of a completely different appearance and psyche. Performing slightly more drastic modifications, we can perform a transformation of mister XY into lady XX. Going further, we can transform her in turn into something else, for instance a creature from $\mathrm{X}$ files.

Summing up all the said above, mister XY does not exist as an absolutely determined object of a clearly defined identity or fact (or even process) of the world, either in the material or in psychical sense. When decomposing our "patient" physically, we also decompose him semantically. The act of bringing XY from ontological non-existence to irrefutable and absolute exis- tence is a magical trick of language. This person appears to be only some loosely, intuitively and roughly determined, "fuzzy" set of states. Shortly speaking, in reality mister XY simply does not exist in a sharp and absolute way.

\section{Language and Facts-Biological Species}

Mister XY is undoubtedly an individual entity. What about general entities, for instance the category "man", which mister $\mathrm{XY}$ in our conviction undoubtedly belongs to? Can we refuse such categories to have a status of absolute and autonomic existence? Undoubtedly we can, even in a more definite and justified way than for individual beings. First, the man originated in the course of biological evolution from an ape (quite recently there were found fossils of species called Sahelanthropus tchadensis, probably closely related to the common ancestor of chimpanzee and man). Biological evolution is a continuous process (although it periodically accelerates and slows down) and the point in the sequence of human ancestors, at which a not-man underwent transformation into man cannot be indicated in any non-arbitrary way. It would be like two ape parents suddenly gave birth to a man. This also concerns the origin of (self)consciousness (if we assume, which is not obvious at all, that, for instance, chimpanzee does not yet possess any early form of consciousness). Consciousness was emerging (because it had to) in a continuous way in the course of biological (and later social and cultural) evolution. Secondly, during ontogeny (embryonic and post-embryonic development), a grown manin his biological and, above all, psychic aspect-gradually develops stage by stage from the embryo into a newborn infant, a child and a young man (it is not at all clear whether these two aspects appear in a perfectly synchronized way). According to religious dogma, the absolute beginning of humanity is defined to occur at the moment of fertilization (combination of a sperm and an egg cell and formation of a zygote), at which point egg cell is endowed with a soul. However, fertilization is not an instant "moment", but a multi-step process composed of many events and stretched in time (and therefore, in accordance with the theme of this article, fertilization is not an absolute fact of the world; yet it is seen as such by religion - extremely magical by its very nature). After the first zygote division, the two resulting cells can dissociate and give rise to two one-egg twins. Do the twins have throughout their life one soul or is soul divisible? Rarely, it happens that a zygote is transformed as a result of embryonic development not into a newborn, but into undifferentiated ball of cells. What about the soul and humanity of such a ball? While one can (although it may seem completely fruitless) discuss the biological, structure-functional beginning of a man in the course of ontogenesis, it is obvious that his psychic aspect can emerge only after the formation of functional neurons, and therefore in a rather late phase of embryonic development. Anyway, the human psyche cannot be formed without a rich inflow of sensory stimuli that takes place only after birth. Certainly, an adult chimpanzee possesses a much richer mental life (no matter how it is defined) than a fertilized human egg cell. Many stages (especially the initial ones) of the embryonic development of chimpanzee and man are very similar. For example, at a certain stage, the embryo of both species possesses gill clefts and a tail-a remnant inherited after our fish ancestors. The thesis proclaimed by unconditional opponents of abortion that the embryo at this stage is already a man with full rights seems to be difficult to defend, at best. And 
all of this results from the strength of magic dogma-extremely powerful, but artificially created "facts" of the world with claims to the Absolute. Of course, the "soul" constitutes a major example of bringing a delusive entity into existence by creating a language name that corresponds to it.

However, the above examples of lack of good semantic specification of the category "man" (as a general being) are only some of a huge number of kinds of such lack of good specification. We can imagine objects or phenomena that differ from man to any large (or small) extent, with respect to one or many of an uncountable number of properties that characterize the category "man" (I leave here aside the question, whether they would be biologically functional or not). In the present period on our planet, this category seems to be relatively well separated and determined, because there do not exist, here and now, any "entities" very similar to humans (for instance, our immediate ancestors: Homo habilis or the genus Australopithecus). However, this does not mean that such entities could not exist in principle. For instance, it is possible (although the probability is extremely low) to imagine that, on other planets in the Universe there evolved organisms that resembled people very much, but differed from them by various complexes of features. In the multi-dimensional continuum of such possible organisms, it seems to be simply impossible to strictly separate and define the category "man", as it is impossible to strictly (with infinite accuracy) separate purple color on the spectrum of colors, continuous in its essence (although the latter seems to be a great deal easier because of the uni-dimensionality of the difference).

Therefore, during analysis of general entities and categories, it appears again that they are created by the magic of language, in this case the magic of the names "designating" such entities. However, this designation is only apparent in this sense that the designated categories exist at best in a very fuzzy, approximate, intuitive and not strictly determined way, while at worst, they are simply delusive. Many philosophers postulated that different sets, for instant the set of red cars, exist in an objective and real manner, independent of our minds. Of course, from the point of view represented in the present article, such sets are entirely products of the mind, and in particular of language that in a magical way attributes absolute existence to "facts" designated by its names.

\section{Language and Facts-Physical Objects}

A similar analysis can be carried out in relation to biological entities devoid of consciousness as well as inanimate entities. This concerns both given concrete objects (a given concrete apple, planet Earth, stone) and categories of objects (apple as a sort of fruits, planet as a category of astronomical objects). I think that the Reader will have no problems with transferring the above reasoning carried out in relation to mister XY or man as a species to the mentioned objects/categories. Anyway, the paradox of the heap is widely known in the form related to physical objects (if somebody takes sand grains from a heap one by one, it is not possible to determine strictly at which point the heap ceases to exist, i.e. to be a heap). It can be mentioned as a curiosity that quite recently Pluto was deprived of the status of a planet by a scientific committee, although this decision was not undertaken unanimously, and the assumed criteria arose controversies. Fuzzy logic is frequently applied to tackle the problem of the vagueness of language.
A more serious problem is met in the case of such indivisible (at least at the present stage of knowledge and scientific theories) objects as elementary particles, for instance photon, electron or one of a few distinguished kinds of quarks. Because they are indivisible, one cannot take away their elements from them. All electrons, for instance, are "identical" (have the same properties, e.g., electrical charge, resting mass, spin or magnetic moment). Therefore, it seems easy to distinguish the category of objects called "electrons" and to indicate univocally a given particular electron. However, at a closer look, there appear serious difficulties. Particular electrons occupy different places in space and, therefore, it must not be said - in agreement with the real state of things - that all properties of particular electrons are the same (there are different space coordinates of their location, different intensities of the electrical and gravitational field in their neighborhood, different locations of other elementary particles). Electrons can be created or annihilated in various quantum transformations, in which other elementary particles also participate. Once a conception appeared that all electrons are in fact multiplied "reflections" of one electron (while positrons - electron anti-particles - are electrons travelling back in time) (Coveney \& Highfield, 1991). However, this conception seems now to be much outdated (it required to assume that there exists the same amount of matter and anti-matter) and citing it here and now could seem unfair. So let us go further on. Moving electrons have greater mass than resting electrons (therefore mass depends on the velocity in relations to other objects). We do not understand in fact what the electron is. According to (standard) quantum mechanics, electrons behave both as waves and as point particles. In some contemporary conceptions (string theory), the electron is not a point particle, but a circular string vibrating in different ways. However, we have no idea (and, on principle we cannot know this, because of the nature of the "content" of our mind that is different from the nature of the "content" of the Universe), what could be the "substance" of such a point or string (here we crash in our cognition against the so-called hard problem of matter, irresolvable in its essence). Before it is detected, an electron is neither a point nor a string, but it propagates in space as a wave. Therefore, the above statement about its determined location was already an abuse. An electron bound in an atom is not located in any particular place, but forms a kind of a "cloud" (that can have different shapes) called orbital, whose local "density" determines the probability of finding a given electron at different points in space. As a wave, a free electron can pass through two slits simultaneously, and its location and velocity are generally determined only with a certain approximation. The more exactly we know the location, the less information we have about the velocity (impetus), and vice versa (the Heisenberg indeterminacy principle). But this happens only until the moment of an electron is detected by some apparatus, when there takes place the so-called reduction of the wave function and the electron becomes localized in a particular place in space. Nobody has in fact an idea, what such a reduction consists in. The so-called conception of decoherence says that the stronger an electron interacts with the surroundings, the more it loses its wave properties, the less it is "blurred" in space (Penrose, 1990). Therefore, the properties of elementary particles do not belong to the particles themselves, because they are also derivatives of other "objects". Two elementary particles-for instance two photons or a pair electron-positron that originate together as a result of certain quantum processes - constitute to some extent 
one whole (so-called quantum entanglement) (Tegmark \& Wheeler, 2003). This means that the properties of these particles are strictly connected, although none of these particles taken separately has these properties determined. If such particles go away from each other in opposite directions and find themselves at a distance of, say, one million light years, then determining such properties (by measurement) for one particle determines automatically and instantly the properties of the other particle (for instance, if a certain property adopts the value of 1 for one particle, then it adopts the value of -1 for the other particle). In other words, particles (their properties) are correlated-this constitutes a manifestation of the so-called nonlocality of quantum mechanics (it has been confirmed experimentally) (Tegmark \& Wheeler, 2003). It is worth to emphasize that in the past of the Universe, in particular just after the Big Bang, when the Universe was very small, pairs of elementary particles repeatedly underwent creation and annihilation, and therefore we have reasons to suppose that all of them are mutually correlated with each other. And in the face of this, the properties of elementary particles are determined by the context of all other elementary particles. Therefore, something like a single, isolated elementary particle simply does not and cannot exist! In fact, the problem is even more enigmatic. This is so because, at least according to some theories, the positive energy related to matter counterbalances exactly the negative energy resulting from the distance in space between bodies endowed with mass in the gravitational field, and therefore the total energy of the Universe is exactly zero (Hawking, 1988). Therefore, it can be said that, after the Big Bang, there originated only information (related to negentropy) about mutual separation of matter and space. In the face of this, the existence of matter - and therefore also of elementary particles - would be dependent on the existence of space. And ultimately, the existence of anything in the Universe would be dependent on the existence of anything else. Therefore, for instance, an electron is not a fully autonomous object (or a category of objects) that can be considered and analyzed separately from other objects, and to which univocal properties and independent existence can be attributed. Therefore, we deal here, at least to some extent, with a not fully determined notion, with a magical name, which makes the pretense of absolute and independent existence of electrons of determined, univocal and understandable properties.

Up to this point, I have been discussing mostly "objects" in the real world, but the same reasoning also applies to processes vs. "events". There is no "discrete" causality in the real world, where a "sharply-defined" reason causes a "sharply-defined" result (an "event"), for instance, in the case of snooker balls, when one ball strikes another on and sets it in motion. Instead, there is only continuity of processes, for instance, when some atoms of one ball, kept together by the electrical force, interact with some atoms of another ball, and the latter moves as a whole, also because the electrical force that keeps its atoms together. In fact, one should talk not about atoms, but about electron coats of atoms (orbitals) that are "blurred" in space, as discussed above. In this way, macroscopic "facts" are extracted by the human mind (not only according to language, but also the mechanism of sensory data integration by the brain) from microscopic continuous processes.

Shortly speaking, when we have a name, it seems to us that we know what the electron is, we "domesticate" the mystery that surrounds it. At the same time, it should be remembered that (as discussed above) the names and notions developed within the frame of formal and natural sciences correspond still much better to various aspects and "objects" of the real world and they "adhere" much better to the external reality than those existing for instance within religion or (most of) philosophy. For this reason, they are much less magical.

\section{Language and Facts-Logic and Mathematics}

What about the languages of mathematics and logic, apparently the most strict and unequivocal of all languages? Are they not - through their strictness and unambiguity-completely nonmagical, perfectly coherent and "objective" (devoid of accidental and subjective "contaminations")? Do they not-through sharply defined, discrete objects and rules - describe the world in an absolutely certain way, devoid of any doubts? Well, it depends on how one looks at this problem. Many mathematicians and logicians believe that their disciplines and objects they deal with (such as logical syllogisms, numbers, sets, functions, various kinds of arithmetic and geometry) exist independently of the material reality and human mind, in the world of ideal Platonic entities. However, if somebody thinks (as I do) that the Platonic world is an illusion, an empty name, then the language of logic and mathematics would be (in a sense at least) the most magical of the languages known to us, as it brings into existence entire independent universes. Let us start with logic. Its rules seem to be obvious and even trivial. For instance, the law of transitiveness of identity that says: "if A is identical with $\mathrm{B}$ and $\mathrm{B}$ is identical with $\mathrm{C}$, then $\mathrm{A}$ is identical with C", or the postulate saying that the sentence "the quantity (number) $\mathrm{B}$ of A is located within C" is either true or false. So far, everything seems to be all right. The problem begins at the moment, when one starts to substitute real or thinkable objects for A, B and C. Let us consider the sentence "A is identical with B". What does it mean "identical"? If one apple differs from another by at least one atom, is it identical with it or not? And if the atomic composition is actually the same, does the spatial location still not make a difference? After all, such a difference means a different situation in relation to various objects, different gravitational and electrical field intensity, different quanta of electromagnetic radiation (photons) reaching the surface of an apple and exciting atoms entering into its composition, and so on. Anyway, the notion of "identity" of two apples does not make any sense, because of quantum indeterminacy. It allows for an infinite number of combinations of locations of particular atoms and elementary particles in apples. Quantum mechanics is statistical in its essence and therefore two apples on principle cannot be identical! As it is discussed above, the property of identity cannot be applied even to single elementary particles, including those entering into the composition of apples under consideration. And therefore, the above-quoted, apparently innocent sentence is in fact nonsensical, simply impossible to be correctly and logically formulated. The situation described by the analyzed statement can occur only thanks to the magic of language.

Let us take another sentence: "there are two apples in this basket" (being an exemplification of the general sentence "the number B of objects A is located within object C"). According to the analysis carried out above, neither apples nor baskets can be identified in an absolute way. Therefore, this sentence can be neither unconditionally true nor totally false (and so, it cannot adopt the logical value of exactly 1 or 0 , but only some intermediate value). Similarly, it is impossible to define sharply the 
relation of "being located within". For, what can one do with the situation, when one atom protrudes beyond a basket? Finally, let us consider the number "two", especially that it refers directly to mathematics. Since there do not exist sharply defined objects, numbers have nothing to count in the real world, while the Platonic world is in my opinion (as I already mentioned) a delusion of our mind, caused by the "fact-creating" magic of language. Generally, logico-mathematical objects and relations constitute elements of the physical world that are extracted, sublimated and arranged in various combinations (compare e.g., Barrow, 1992 for discussion). Thus, as the number "two" was extracted from two apples, two goats and so on, so a tight string was the prototype of a straight line and two sticks crossed at a possibly large angle gave rise to the notion of the straight angle. Piaget demonstrated how children acquire understanding of mathematics, when they gradually pass from concrete objects and facts to abstractions and symbols (Piaget, 1953). It is likely that mathematics developed in a similar way during biological and social evolution of humans. Of course, contemporary mathematics deals with much more complex and subtle "objects" than a number or a straight line. There is also no "one mathematics": within mathematics, there coexist various competitive (alternative) constructs, such as Euclidean geometry and countless plethora of non-Euclidean geometries. There exist many alternative kinds of logic. Most of these varieties of mathematics and logic seems to have little to do with the real world, although some can appear to be very useful in describing certain aspects of the physical reality. However, in my opinion, all of them are a result of arranging conceptual and linguistic blocks into different combinations, while the blocks themselves are taken more or less directly from everyday experience, and also from neural structures in the brain that process signals coming from receptors and carry out autonomous associations on a higher level (broadly understood thinking processes - see Korzeniewski, 2010). Similarly, the same blocksatoms and molecules - can be potentially arranged in various configurations corresponding to functional living organisms ${ }^{2}$ that do not appear on Earth. This would be equivalent to creation of "alternative life" (after all, most probably some of its possible forms exist on other planets in the Universe). However, this does not mean that such "potential" living organisms belong to a certain Platonic world of biological entities. Additionally, I suspect that at least some (if not all) mathematical concepts extracted by mankind are internally contradictory-this concerns for instance the concept of infinity (also infinitely small points). In fact, how is it possible that infinite number of points multiplied by infinitely small (zero) size of a point gives three centimeters in one case and five centimeters in another case? (To be sure, the great majority of mathematicians regard this problem as overcome for a long time; being aware of this, I take the liberty of disagreeing with them). The Cantorian conception of infinity (commonly accepted now) says that the numerical force of integer numbers is equal to the numerical force of even numbers (both sets have the same cardinality), because they can be univocally attributed to each other in pairs, e.g., 1 2, 2 - 4, 3 - 6, $4-8 \ldots$ and so on ad infinitum (see e.g., Penrose, 1990). However, in a certain important sense, there are more integer numbers than even numbers, because this is true for any finite sequence of numbers longer than one number. In other words, in any finite (and infinite) sequence of integer numbers,

${ }^{2}$ Functional biological systems would correspond to non-contradictory and consistent mathematical systems. integer numbers are "more densely packed" than even numbers. This statement also applies to the same degree, for instance, to integer numbers and their squares or cubes. Therefore, the Cantorian conception of infinity and equinumerosity is only one of possibilities, and its common acceptance constitutes, to some extent, a manifestation of a certain intellectual fashion. Generally speaking, I suspect that the concept of infinity is internally contradictory, or even nonsensical. It was started by a simple word-concept conglomerate, for instance, "if we continue to add number 1 to a sequence of subsequent natural numbers, we will never terminate" or "if we continue to divide a segment ad infinitum, we will come to points of infinitely small (zero) size". However, this "operational infinity" does not imply any transcendent "real infinity". Considering the above stated, absolute mathematical facts are not so "absolute" (see e.g., Barrow, 1992). The name "set of all sets" seems to be strictly and precisely defined, easily and intuitively understood. However, it appears that it is an empty and nonsensical name, as such a set cannot exist, because it has been proven that the set of all sub-sets of a certain set is bigger than this set itself, and of course no set can be greater than the set of all sets. By the way, in my opinion, for a large part of mathematicians and logicians, their discipline constitutes a substitute of religion as a certain Absolute, existing somewhere in the (Platonic) underworld. Though, of course, I prefer much the linguistic magic of mathematics and logic than the linguistic magic of religion.

\section{Language and Conceptual Network}

So far, the relations between language and the external world, "objective" reality have been discussed. However, it should be emphasized that, in my opinion, language does not refer directly to the world. Something - which I call conceptual network and the neural network underlying it - mediates in the relations between them. As I proposed in detail earlier (Korzeniewski, 2010; Korzeniewski, 2013), the "substance" of our psyche is a network of concepts being a mental correlate of the network of functional connections between neural cells in the brain. This network develops during lifetime through extension of the already existing system of connections caused by an inflow of signals from receptors (sensory impressions) and as a result of its autonomous activity (processes of thinking). In turn, the conceptual/neuronal network itself serves as an interpreter of incoming sensations that enables their understanding, and also manifests the above-mentioned autonomic activity (again, associated with understanding of carried out neural/mental operations). In the process of individual development (ontogenesis), appropriately directed and organized cognitive processes (whose general mechanisms are inborn and therefore shaped by biological evolution) lead to an approximate representation (sometimes better, sometimes worse) of certain aspects of the external world within the neural/conceptual network, especially these aspects that are important for humans as biological and social beings. To put it metaphorically, the neu$\mathrm{ral} /$ conceptual network entwines the reality in such a way that particular mesh holes correspond to various aspects of the world (like the spider web entwines the surface of a stone sculpture; however, while the spider web is two-dimensional, then the conceptual network has a potentially unlimited number of (semantic) dimensions) ${ }^{3}$. When (either during individual development or biological evolution) language - being a special part of the conceptual network in this sense that it facilitates effective use of this network as a whole and does so through 
attributing discrete names to concepts (I leave aside here the obvious function of interpersonal communication) - enters the stage, some aspects of the world, represented by mesh holes, become the above-discussed "facts" of the world. Language names do not mean by themselves (this would be on principle impossible), but through concepts (and therefore-fragments of the neural network) that underlie them (Korzeniewski, 2010; Korzeniewski, 2013). Concepts, in turn, mean through relations with other concepts (meaning by connotation). It is just the conceptual "lining" of language in the mind (brain) that allows us to understand language, while a computer does not understand it, while also operating on different forms of language, yet not equipped with the underlying conceptual network.

For this reason, the human thinking is on principle not algorithmic. In my opinion, any discussions whether thinking is algorithmic or not (see e.g., Penrose, 1990) are pointless. An elementary knowledge about the functioning of neurons and their groups is sufficient to conclude that they work in a continuous, analogical and therefore non-algorithmic way. For instance, the frequency of impulses conducted by axons or the concentration of neurotransmitter in the synaptic cleft can adopt one of a continuous spectrum of values. Thinking is based primarily on the conceptual network, and not on linguistic names and formulas. Therefore, the algorithmic thinking is one more apparent "fact" created by the magic of language.

The correspondence between linguistic names and "facts" of the world is a two-step relation-the conceptual network "winds around" (various aspects of) the world, while the names of language are assigned to those elements of the conceptual network-concepts-that are best separated, determined and characterized by the greatest "intensity of the semantic field". In other words, language realizes its meaning in relation to the world through the conceptual network. Granting names to concepts, on the one hand, considerably facilitates manipulation of concepts ${ }^{4}$, their ordering in grammatical and logical structures. On the other hand, however, it causes a far-going absolutization of these concepts, their transformation into discrete and apparently independent entities (since something continuous, fuzzy, partly undetermined is transformed into something apparently unitary, discrete, self-dependent, sharply separated). The same happens of course to the aspects of the world represented by these concepts. The former suddenly undergo a conversion from entities connected by interactions with other entities into autonomous, absolute and sharp "facts" of the world. This is a manifestation of the duality of language, which enables a more efficient formation and use of the picture of the world based on concepts, but at the same time, it co-shapes, co-determines, deforms and even distorts this picture. Of course, the discussed problem of the "world-creating" role of language concerns not only the discreteness of linguistic names and bringing simple facts into existence, but also grammatical rules, through which sentences of language impose their structure on the external reality, order it (or in fact its picture in the brain and mind) into specific forms, depending on the kind of this structure.

In a certain important sense, the conceptual network is a phenomenon superior to language, as it is more primary and

${ }^{3}$ The analogy with the spider web demonstrates also why it is impossible to resolve the hard problem of self-consciousness: as the substance of the spider web is completely different from the substance of the sculpture, so the substance of the conceptual network (psyche) is totally different from the substance of the external world.

${ }^{4}$ Therefore, my idea resembles to some extent Fodor's language-of-thought hypothesis (Fodor, 1975). spacious than language. As I mentioned, the conceptual network in humans constitutes the semantic "scaffolding" of language, without which the latter cannot exist, and first of all possess meanings. In animals, the conceptual network (much simpler than in humans) is devoid of the linguistic "superstructure" (this is probably one of the reasons limiting the complexity of this network). Besides, as I stated above, even in humans, linguistic names are attributed only to the best separated and determined concepts (what anyway strongly stimulates their further individualization and development). Therefore, large areas of the conceptual network-composed of barely sketched concepts, allusions about senses - do not find equivalents in the sphere of language.

This indirectness of the relation of correspondence between names of language and "facts" (caused by the conceptual network) leads to a large divergence between the world and its linguistic picture or representation. This results from a double incoherence (or rather very imperfect coherence): between the conceptual network and the external world as well as between language and conceptual network. In the face of this, it is really astonishing, how deeply and universally we are convinced (frequently implicitly) of the existence of "sharp", clearly separated objects, processes and categories within the "objective" reality. The conviction results, of course, from the way of functioning of human brain and mind, the way that is shaped by biological evolution. Language constitutes so effective a tool of interpersonal communication and manipulation with the conceptual network in thought processes, that it pays to tolerate its "fact-creating" or even absolutistic "side effects". Because, the primary aim of biological evolution was not to perfect "sublimated", abstract ("philosophical") cognitive abilities, but to improve purely instrumental functioning of humans in their physical-biological and socio-cultural environment. Languagebeing a very efficient tool helpful in achieving this purposeshaped at an occasion the mind in such a way that it has a strong predilection to quantize the continuous in its nature environment. Additionally, language was becoming a more and more autonomous phenomenon and started to create constructs (names, messages) that had little, or even nothing to do with the external reality. In simplest and most trivial cases, this consisted in elementary combinations of "facts" to create new "facts" on this basis. Thus, a combination of a man with a bird gave an angel, while that with a lion produced a sphinx. More abstract and complex operations of this type led to tearing off the mental sphere from the corporal (material) sphere, which gave origin to astral bodies or the immortal soul (spirit). Big ontological or religious systems are most "advanced" in this combinatorics of linguistic elements, which also makes them leaders in getting completely detached from the real world.

Simply speaking, after gaining a certain autonomy, language - that initially originated in order to (among others) represent the conceptual network, and thus the external world, which was greatly facilitated by the combinatorics of language elements (names) based on grammar-began to experiment with such combinations of elements that did not have any real equivalents, as angels mentioned above. What's more, it was able to impose these constructs onto the conceptual network, i.e. the "carrier" of senses (meanings). Thus, it forced in a sense the belief in the existence of "designates" of such combinations in the external world. In no different way did there originate big religious and philosophical systems. Of course, I perform a purposeful language subjectivization. In reality, we deal with 
impersonal processes occurring in fragments of the conceptual network lying at the base of language. I also leave aside the mechanisms responsible for the generation, selection, estimation and acceptance as well as cultural consolidation of such-and-not-other combinations of language elements, not to mention yearnings, fears, needs and pure chance. I also do not deny that such deceitful (in the cognitive sense) activity can have some adaptive value. For instance, a certain mythology can be useful tool that integrates a society or tribe. Yet, all these matters, undoubtedly important, do not change the role of language in the creation (through free element combinatorics) of delusive entities and that role is what I am focused on after.

When language entered into primitive human societies, it was accompanied with such fare-dodger phenomena as the system of arbitrary cultural senses, mythology, religion or just magic (in the narrow sense). Therefore, the magic of language creates not only discrete facts in the continuous real world, forces not-well-determined aspects of the reality into a strict corset of linguistic names, but it also brings into existence mental phenomena that do not correspond to nothing in this reality, namely spirits of ancestors, supernatural powers, various gods, rituals that are to gain their favor (prayers, scarification of animals or humans) and so on. And all this happens because of the pragmatic and opportunistic biological evolution that shaped our brains for hunting mammoths and achieving social success (a handy tool for propagating our genes), and not for faithful cognition of the world that is not contaminated by anything.

\section{Language and Self-Consciousness}

According to my conception (more comprehensively discussed before: Korzeniewski 2010, Korzeniewski 2013), consciousness in the psychical sense and self-consciousness, that is "feeling" of one's own "I" (ego) emerge as a result of selfdirectioning of the cognitive "center" in the human brain on itself. This self-orientation would consist in the fact, that, apart from receiving signals from the external world (processed by sensory centers), this center begins also to receive signals from itself. In this way, it creates within itself a model of itself, projects its own picture in itself. In other words, we deal with here with a recurrent reference to itself. It seems likely that it is not a matter of coincidence that the only creature known to us endowed with (self)consciousness - man-possesses a highly developed language. In my opinion, in the process of biological evolution (but also individual development), language constitutes a good candidate, if not for as much as the source (generator) of (self-)consciousness, then at least for a catalyst of its development. Language - as it is also, after all, an element of the conceptual network - facilitates greatly the self-referring relation between this network and itself, the process of modeling itself within itself, the process of entering a certain meta-level and adjudicating also about itself (one can notice here an analogy to famous logical paradoxes: the liar's paradox, Russell's antinomy of classes (Penrose, 1990; Barrow, 1992) and Gödel's proof (Penrose, 1990), which are also based on the relation of self-reference). Language is after all a sort of a model of the conceptual network. Its names correspond, as I mentioned above, to the best separated concepts. A very important role is played here by the efficiency of language in handling the conceptual network, and also by the formal structure of language that enables it to refer to itself (an therefore in fact to handle and manipulate itself) as well as to separate sharply (through naming) a certain set of phenomena or processes. This is the origin of one of the most delusive facts of the world (this time the "internal" one): our absolute, indivisible and invariable "I" (ego) that decides about our identity, or even essence 5 . From here, it is not far to the immortal soul (spirit), underworlds that this soul inhabits and similar delusions.

Something like one absolute, indivisible and invariable egothe quintessence of the identity of our being - simply does not exist. As this contradicts our everyday subjective feeling and common opinions, I will devote a few words to this problem. One should start from the fact that the "I", understood as (self) consciousness, is not separated from "non-I" by any absolute border. It emerged in the course of biological evolution of man and emerges each time from psychical non-existence during development from a fertilized egg cell to an adult individual. By the way, there are reasons to think that in both cases the development of ego proceeds in parallel with the development of language and both these processes mutually drive (stimulate) each other, in accordance with the conception presented above. For instance, let us consider a known phenomenon of the so-called infantile amnesia that consists in the fact that a child has no (conscious) memories preceding the period before he/ she masters language. Secondly, the "content" of the "I"- and therefore, both the momentary content and general features of psyche - change in the course of human life. A man learns, gathers experiences, his views evolve, he acquires new memory traces, while many old ones undergo obliteration. Even if we abstract from the infant period, then an old man certainly is not "the same man" as the young man from whom he has developed. The only thing that connects them is a certain continuity (smaller or greater) of the evolution of the traits of psyche (memory traces of course play here an important role). However, much more drastic changes are possible. Personality changes significantly in a short period of alcoholic stupor or drugged state. Anyway, it is enough to recall that the much more usual shifting of attention from one thing to another or turning consciousness off during dream. Long-term and irreversible changes (most frequently damage and impoverishment) of psyche are caused by diseases, like Alzheimer or Parkinson disease, as well as by vast damages of the brain inflicted by an accident or stroke. A frequently cited example is callotomy (section of corpus callosum that connects both brain hemispheres), leading to the emergence of two almost completely independent selves (each of which possesses its own "free will") that inherit some properties of the initial self, although they are undoubtedly impoverished compared to the original self. Contrary to all appearances, the psyche is not indivisible either. It consists of many "parts" and aspects that can be selectively handicapped as a result of a damage inflicted on various brain areas. Therefore, this is another case, when language creates an apparently absolute fact of the world, namely our own invariable and indivisible "I", which is in fact an evolving complex of processes that constitute the base of (the content of) our (self)consciousness and that can be determined and separated only in an approximate and fuzzy way.

\footnotetext{
${ }^{5}$ I will add for clarity that I do not negate the emergence of the (epi) phenomenon of (self)consciousness from a certain form of brain functioning, as I do not deny the emergence of the phenomenon of life as a result of a certain functional organization of matter. I only put in doubt the existence of an absolute, unchangeable and indivisible "I" (or soul, spirit, etc.), by analogy to the negation of an absolute existence of a fact of the world in the form of mister XY.
} 


\section{B. KORZENIEWSKI}

\section{Language, Science, Philosophy, Religion and Magic}

Due to the lack of perfect adherence of both language to conceptual network and of the network itself to the world, language can describe the real world only in an approximate way. Additionally, due to absence of appropriate methodology, the adherence of language to the world is much worse in philosophy than, for instance, in science (such disciplines as, for instance, religion represent the pure magic of language, practically uncontaminated in its essence). Already in science itself, and in trials of unification of whole physics in particular, our conceptual apparatus as well as language (especially mathematical language) seem to face huge obstacles in their attempts at representing the reality. Philosophy exhausted the majority of its potential to describe the real world hundreds, if not thousands years ago. In fact, during the last centuries, the only significant progress has been made within the framework of sceptical, analytical philosophy (e.g., Hume, Kant) that underscored limitations of our knowledge of the world. Contemporary huge developments in neurophysiology also indicate univocally that humans are not universal cognitive machines. Moreover, man has an intrinsic inclination to create concepts referring to delusive entities and to do so for his own use. Such concepts undergo unusual enhancement through attributing appropriate linguistic names to them. This process took place in different sorts of mythologies, religions, astrology, alchemy, masonry, and generally-according to the viewpoint presented here-in broadly understood magic. In all these social phenomena, an important role was played by proper names, incantations, spells, rituals and ceremonies. All of them generated the sense of initiation, esoteric knowledge, of belonging to an exclusive group of the elect. All beguiled with access to the Deepest and Ultimate (and frequently also Inconceivable) Mystery. The same also applies to a large extent to philosophy (especially ontology) which operates with highly delusive names and concepts that do not correspond to anything (or almost anything) sensible. These concepts are predominantly simply senseless and very poorly defined at best. Here belong the concepts of the spirit (soul), matter, predicate, monads, causality, fact of the world and many, many others. Philosophy also lays claims to the absolute truth. The higher the level of abstraction is reached by philosophical reflection, the less it has in common with the real world, the more it is devoid of any sensible content, the more it can be defined as juggling with meaningless names. Therefore, the most reasonable philosophy is the one that is directly based on contemporary achievements of science and that integrates and interprets them on a current basis.

For this reason, if we undertake to engage in a philosophical dispute, we ought to know exactly, when this dispute should be terminated. Otherwise, it simply boils down to futile, terminological (linguistic) "mumbo jumbo". In other words, one should avoid entering such abstract and "transcendent" peaks of philosophical reflection, where any statements appear to be only a pure phrase-mongering. Somebody who professes solipsism or, oppositely, denies real existence of consciousness does not understand that the concepts of the spirit (soul) and matter (functioning quite well in everyday life) have no well determined meaning in philosophy. Therefore, instead of assuming absolute, magical existence of names and concepts (and facts), it is better to retain a certain minimum of common sense and mark out a conventional line, beyond which it makes no sense to continue philosophical disputes. Of course, setting such a line is not easy and nobody can lay exclusive claims to know its course. Besides, it is easy (I know this from my own experience) to go too far in the fervor of discussion and cross the borders set by oneself. Therefore, it is important to respect them consistently. Finally, it is worth mentioning that (again, I judge by myself) the awareness of the existence (and liquidity) of such a border is formed (and evolves) in time, together with an ongoing analysis of the nature of science, philosophy and all that lies in between.

It must be honestly admitted that science is by no means free of magical admixtures. For instance, physicists are inclined to attribute an excessive degree of reality to mathematical objects that are to describe the real world. Biologists believe (to a smaller or greater extent) in the existence of living individuals and species. Astronomers believe in the absolute existence of stars, planets, and so on. I mentioned this above. Science, however, is nonetheless the least magical of all disciplines of human intellectual activity. Because only science possesses a reliable methodology (including, first of all, experimental tests and observations), which allows a relatively good (although by no means ideal) attribution of linguistic names (including those of the language of mathematics) to various aspects and objects of the real world. Therefore, it tries to describe the world and not to create it. Yet, it is only partly successful in fulfilling this task.

Coming back to the magical aspects of philosophy, I would like to mention yet another issue, namely that of words-master keys, as I call them. These are names that are in principle empty, yet they are willingly used in philosophical disputes as they make the impression of wise and profound concepts. I will give one example. Neurophysiologists (as well as researches from kin disciplines) for decades carry out laborious studies and build theoretical models that are to reveal how (for which kind of functional matter organization) self-consciousness (psychical sphere) emerges from the functioning of the neural network in the human brain (the so-called soft problem of consciousness). Philosophers, in turn, in spite of their frequently week understanding of neurophysiology, resolve the problem by saying that the mind cannot emerge from the functioning of matter, because both entities are "incommensurable". Similarly, nineteenth century philosophers denied the possibility that life can emerge from inanimate matter, because they were unable to accept the fact that life is only a set of atoms organized in a special way ${ }^{6}$ and there is no "vital force" behind it. Huge progress of biology in the twentieth century proved this view to be completely false. Generally, human brain shaped by biological evolution (this concerns not only philosophers, but also, for instance, physicists and biologists) has huge difficulties with direct "seeing" and comprehending the phenomenon of complexity and also with the consequences resulting from it, including emergence of phenomena and properties on a higher level of complexity hierarchy from interactions of elements on a lower level. This applies not only to such phenomena as life or consciousness. We are even unable to explain in detail, why huge numbers of complexes of two hydrogen atoms or of two oxygen atoms take the form of gases, while huge numbers of complexes of two hydrogen atoms and one oxygen atom take the form of a liquid, water, of such-and-not-other properties.

${ }^{6} \mathrm{I}$ am not a primitive reductionist, as I believe that the phenomenon of complexity exists objectively-see below. 
A leading example of magical thinking in philosophy is found in the attribution of real existence to universals (general beings like sets, categories or notions). This is the so-called philosophical realism. In my opinion, however, the problem turns out to be completely futile, when one realizes that they are simply concepts created in our brain/mind by common neurophysiological processes that underlie brain functioning (they correspond to what I call associative structures-see refs. Korzeniewski, 2010; Korzeniewski, 2013).

While in science the admixture of the magic of language is relatively small (thanks to its relatively reliable methodology), it constitutes the dominant element in (abstract "positive") philosophy (especially ontology), not to mention religion. On the other hand, science frequently suffers from excessive specialization and lack of a general approach. Therefore, it can be said-simplifying the matter somewhat facetiously-that philosophers know nothing about everything, while scientists know everything about nothing. This means that philosophers have a wide, integrative view, but they lack effective methodology allowing for a relatively reliable cognition of the world, while scientists have such methodology at their disposal, but their interests are very frequently limited to very narrow segments of the reality. I think that one ought to try to reconcile these opposite approaches to cognition of the world and to gain a possibly integrated picture of its various aspects (the domain of philosophy), directly based on the relatively "hard" knowledge, acquired thanks to a large contribution of empiricism (this is what science deals with).

\section{Language and Ethics}

One of the most blatant cases of the magical, fact-creating power of language is fundamentalist ethics. An example of such thinking is found in the unconditional prohibition of abortion, based, among others, on the dogma that a zygote is already a human with full rights, endowed by God with the immortal soul at the moment of fertilization. Fundamentalist ethics views all possible moral conflicts in terms of zero-or-one solutions and grants an absolute status to the laws it chooses to preach. However, psychic and social life is of course too complex to be unequivocally codified, forced into a corset of simple, unshakable rules that do not contradict each other. This was experienced by everybody, who faced the so-called moral conflicts, that is by all normal people. Therefore, any attempt at constructing absolutist ethics simply takes the easy way out and releases man from the duty of thinking and hesitation before taking a decision, and generally from elementary human responsibility. A ruthlessly consequent realization of literally sketched moral rules leads very frequently to gruesome results. This would not happen if we do not use the magic of language to promote the frequently conflicting motivations of fair and honest behavior to the status of facts (ethical this time).

\section{Conclusion}

My point of view is decidedly relationist, because I do not believe in (the sense of) an absolute existence of (sharp, perfectly determined) linguistic elements, concepts and "facts" of the world (objects, processes, phenomena, categories). I think that both elements of the neural and conceptual network as well as those of the external reality mean by connotation, by being related to each other. The semantic relation of denotationfacts of the world-concepts in the conceptual network-language and its elements (words, sentences) - is very imperfect. Therefore, the picture or representation of (different aspects of) the world in the mental and linguistic sphere is very approximate and "fuzzy". Additionally, it is considerably deformed by the discrete and "stiff" nature of language elements (words, sentences, grammar).

The process of separating and extracting discrete objects from the essentially continuous world (and continuous stream of sensory data) starts already on a pre-linguistic level, at the stage of processing signals from receptors by the sensory cortex (Korzeniewski, 2010, Korzeniewski, 2013). However, language strongly enhances the separation of "facts" of the world, which leads to their absolutization and apparent autonomy.

Through "quantized", discrete nature of its names and sentences built of them and governed by rigorous laws of grammar, language imposes on us irresistibly an urge to classify and categorize everything, to create sharp, absolute facts within the essentially continuous and relational world. Because of this, it stimulated the creation not only of these facts, but also of sets, classes, all sorts of systematics, classifications, hierarchical organizations, divisions into opposites, and so on. This gave rise to our fondness of order, arrangement of elements of the world into simple and understandable constructions, symmetrical and clear-cut patterns, stiff and incontestable rules. Paradoxically, this affection to a simple, universal and preferably absolute and the only possible order gave origin both to astrology, alchemy, magic, philosophy and religion, on the one hand, and to science, on the other hand. The main difference consisted here in the fact that the former drew mainly or exclusively from the creativity of our mind channeled by language and based only on its own inventions, wishes and fears, while science gave preference not to unlimited speculations of "pure mind", but to empirical knowledge, that is to consequent investigation of the external world and to continuous confrontation of the ideas of the mind with observations and experiments. This cognitive modesty allowed scientific reflection to advance step by step, slowly and with many mistakes, yet to reach much farther and do so in a much more reliable way.

Every word, sentence and more complex construct of language is in a certain important sense a magical formula: it creates absolute, "sharp" and discrete entities, facts, objects, persons, laws, sets, processes and categories from nothing, from an essentially continuous spectrum of phenomena, and it fills the real world up with these creations. Naming some entity (giving it a name) in fact brings this entity into existence. It is not so bad if this entity corresponds to some relatively well separated aspect of the world, which takes place in science or everyday life. It is worse if language creates entities or "facts" that are completely (or almost completely) imaginary, delusive, extremely poorly defined or even devoid of any sense, which is the domain of magic, astrology, religion and a large part of philosophy (in particular-ontology). I mean here not only "pure" invention of entities that have no (or almost no) equivalents in reality, like monads or angels, but also more complex linguistic (and, as a consequence, semantic) constructs. General entities corresponding to various sets or categories of elements constitute one of my favorite examples. It seems obvious (at least for me) that such entities exist only as linguistic names (and concepts corresponding to them) in the mind. However, a quite large part of philosophers attribute real, objective existence to them. A good example of inconsistency of constructs based on sets viewed as absolute entities is Russell's antinomy 


\section{B. KORZENIEWSKI}

of classes, which demonstrates that both positive and negative answer to the question "is the set of all sets not being their own elements its own element or not" lead to contradiction. The already mentioned "set of all sets" is a less known, yet equally spectacular case. It appears that this apparently clear linguistic construct that seems understandable without any problems is devoid of any sensible content. Similar linguistic constructs, only apparently filled with real or sensible content, appear in religion. They can take the form of one of the "proofs" of the existence of God (there must exist a most perfect entity, and this by definition is God) or an apparent paradox (whether omnipotent God can create a stone that he could not lift), or something from the Buddhist wisdom (is it possible to clap with one hand). A while of sober reflection leads to a clear realization that these magical formulas constitute simply empty and meaningless juggling with words. Thus, the mentioned disciplines (magic, religion, a large part of philosophy) lead to (magical!) autonomization of linguistic names and phrases, and through this to their detachment from anything real, or even sensible. In fact, can there be something more magical than "The Word became flesh"?

Our thinking (to a large extent) and our interpersonal communication (almost completely) are slaves of language. The problem, however, is that we practically never fully realize this state of affairs. And we necessarily should do so to free ourselves at least partly from the omnipotence of language. When operating with language, one ought to remember about the all the deep and rich subtleties of its relations with conceptual network and external world, and one should not use names in a brute way, believing naively in their absolute and incontestable or even magical status.

The following analogy (simplified out of necessity) can be used to compare language to conceptual network. Language is a fundamentalist, ordered, strictly logical phenomenon that imposes military discipline on our thinking, but also it frequently limits this thinking, reduces it to usual schemes. On the other hand, conceptual network is liberal, even anarchistic, chaotic to some extent, intuitive, full of freedom and inclined to improvisation. The question of which of them should be given priority can be answered only in one way: none. What is needed is the golden mean, such symbiosis of language and conceptual network that enhances their merits and limits their failures. Language in reasoning is a despot and it has by its nature decisive supremacy. Therefore, a great effort is necessary to allow the fertile mists of conceptual network to come to the force. Only ideas first formed in this way should be afterwards exposed and explicitly expressed by dressing them in possibly adequate linguistic constructs.

Of course we will not liberate ourselves from language, and under no circumstances should we do this, because it makes our thinking (use of conceptual network) and communication (exchange of meanings between conceptual networks of different persons) much more efficient. However, we should continuously remember its limitations and faults. First of all, we should be aware that it imposes facts onto the world, facts that consti- tute at best only approximate and imperfect representations of certain aspects of this world, while, at worst, they may have nothing in common with it. Language helps enormously to create the picture of the world, but it also simplifies and deforms significantly this picture. In extreme cases, it brings imaginary worlds into existence. This concerns especially such disciplines of human intellectual activity as religion and philosophy. Therefore, we must be as immune as possible to the magic of language, in order to avoid getting uncritically enchanted.

\section{REFERENCES}

Austin, J. L. (1962). How to do things with words. Oxford: Clarendon Press.

Barrow, J. D. (1992). Pi in the sky. Oxford: Oxford University Press.

Chomsky, N. (1968). Language and mind. New York: Harper and Row.

Coveney, P., \& Highfield, R. (1991) The arrow of time. The quest to solve science's greatest mystery. Flamingo.

Fodor, J. A. (1975). The language of thought. Cambridge, MA: Harvard University Press.

Frege, G. (1960). On sense and reference. In: P. Geach \&, M. Black (Eds.), Translations from the philosophical writings of Gottlob Frege (pp. 56-78). Oxford: Basil Blackwell.

Hawking, S. W. (1988). A brief history of time: From the Big Bang to black holes. New York: Bantam Books.

Kay, P., \& Kempton, W. (1984). What is the Sapir-Whorf hypothesis? American Anthropologist, 86, 65-79. http://dx.doi.org/10.1525/aa.1984.86.1.02a00050

Korzeniewski, B. (2010). From neurons to self-consciousness. How the brain generates the mind. Amherst: Prometheus Books.

Korzeniewski, B. (2013). Formal similarities between cybernetic definition of life and cybernetic model of self-consciousness: Universal definition/model of individual. Open Journal of Philosophy, 3, 314328. http://dx.doi.org/10.4236/ojpp.2013.32049

Penrose, R. (1990). The emperor's new mind. Concerning computers, minds and the laws of physics. London: Vintage.

Piaget, J. (1953). How children form mathematical concepts. Scientific American, 189, 74-79. http://dx.doi.org/10.1038/scientificamerican1153-74

Prigogine, I. \& Stengers, I. (1984). Order out of chaos. London: Heinemann.

Prigogine, I. (1980). From being to becoming. San Francisco: W. H. Freeman.

Quine, W. V. O. (1960). Word and object. Cambridge, MA: MIT Press.

Russell, B. (1923). Vagueness. The Australasian Journal of Psychology and Philosophy, 1, 84-92.

http://web.archive.org/web/20080515181625/cscs.umich.edu/ crshal izi/Russell/vagueness/ http://dx.doi.org/10.1080/00048402308540623

Sapir, E. (1921). Language: An introduction to the study of speech. Brace: Harcourt.

Schrödinger, E. (1992). What is life? Cambridge: Press Sindicate of the University of Cambridge.

Tegmark, M., \& Wheeler, J. A. (2003). 100 years of the quantum mysteries. Scientific American, 284, 68-75.

http://dx.doi.org/10.1038/scientificamerican0201-68

Whorf, B. L. (1940). Science and linguistics. Technology Review, 42, 229-231.

Wittgenstein, L. (1921). Tractatus logico-philosophicus. 